PROFILES OF FAMILY CAREGIVERS PRIOR TO THE DEATH OF A SPOUSE AND THE RISK FOR DEVELOPING PROLONGED GRIEF

L.M. Miller ${ }^{1}$, R.L. Utz, $\mathrm{PhD}^{2}$, K.P. Supiano, PhD, LCSW ${ }^{3}$, D.A. Lund, $\mathrm{PhD}^{4}$, M.S. Caserta, $\mathrm{PhD}^{5}, 1$. University of Utah, College of Nursing, Portland, Oregon, United States, 2. Department of Sociology, University of Utah, Salt Lake City, UT, USA; Center on Aging, University of Utah, Salt Lake City, UT, USA, 3. FT, College of Nursing, University of Utah, Salt Lake City, UT, USA, 4. Department of Sociology, California State University, San Bernardino, CA, USA, 5. College of Nursing, University of Utah, Salt Lake City, UT, USA; Center on Aging, University of Utah, Salt Lake City, UT, USA

The purpose of this study was to characterize distinct risk profiles of family caregivers prior to the death of a spouse/partner in hospice, and determine the extent to which these pre-bereavement profiles helped predict prolonged grief. Using latent class mixture modeling to examine distinct risk profiles among 195 family caregivers, two preloss profiles were identified, one of which was comprised of family caregivers $(n=60 ; 31 \%)$ exhibiting significantly higher anxiety and depressive symptoms, lower active coping scores, less preparedness for the caregiving role, less social support, greater impact on caregiver's health, and more relationship strain between the caregiver and their spouse/partner. Family caregivers who were observed in this higher risk profile were significantly younger, reported lower annual incomes, and were significantly more likely to develop prolonged grief, according to adjusted multivariable models using subsequent bereavement data from 124 participants who remained in the study at 6 to 18 months post-loss.

\section{ASSESSING PRE-DEATH GRIEF FROM A DISTANCE: RELIABILITY OF THE TELEHEALTH-BASED MMCGI-SF}

A. Lindauer ${ }^{1}$, A. Seelye, $\mathrm{PhD}^{2}$, H.H. Dodge, $\mathrm{PhD}^{3}$, N. Mattek, $\mathrm{MPH}^{4}$, K. Mincks, $\mathrm{BA}^{5}$, J.A. Kaye, $\mathrm{MD}^{6}, 1$. Oregon Health \& Science University, Layton Aging and Alzheimer's Disease Center, Portland, Oregon, United States, 2. Minneapolis VA Health Care System, Minneapolis, MN, USA; Oregon Center for Aging \& Technology (ORCATECH), Oregon Health \& Science University, Portland, OR, USA, 3. Oregon Center for Aging \& Technology (ORCATECH), Oregon Health \& Science University, Portland, OR, USA; University of Michigan, Michigan Alzheimer's Disease Center, Ann Arbor, MI, USA, 4. Layton Aging and Alzheimer's Disease Center, Oregon Center for Aging \& Technology (ORCATECH), Oregon Health \& Science University, Portland, OR, USA, 5. Oregon Health \& Science University, Portland, OR, USA, 6. Layton Aging and Alzheimer's Disease Center, Oregon Center for Aging \& Technology (ORCATECH), Oregon Health \& Science University, Portland, OR, USA

Pre-death grief (PDG) is a salient concept for family caregivers for those with Alzheimer's disease and related dementias (ADRD). The prolonged experience of grieving in response to dementia-related decremental losses increases caregiver burden and contributes to complicated grief after the death of the person with ADRD. Therapeutic interventions can ameliorate PDG and measures are available to assess the degree of PDG in caregivers. However, not all caregivers have access to providers who can assess PDG. Behavioral symptoms of dementia and geographic distance are common barriers to caregiver assessment and support. To reduce this barrier, we evaluated the reliability of the Marwit Meuser Caregiver Grief Index Short Form (MMCGI-SF) when administered via telehealth-based videoconferencing. Caregivers $(\mathrm{n}=28)$ completed the MMCGI-SF in person and via videoconferencing. Intraclass correlation between administration sites (in-person versus via videoconferencing) was 0.87 , indicating excellent reliability and suggesting providers can administer the MMCGI-SF via videoconferencing with confidence.

\section{IF WE KNEW THEN WHAT WE KNOW NOW: THE PREPAREDNESS EXPERIENCE PRELOSS AND POSTLOSS FOR DEMENTIA CAREGIVERS}

K. Supiano ${ }^{1}$, M.K. Luptak, PhD, MSW ${ }^{2}$, T. Christian Andersen, PhD, MSW, MS, LCSW ${ }^{3}$, C. Beynon, MSN, $\mathrm{RN}^{4}, 1$. University of Utah, College of Nursing, Salt Lake City, Utah, United States, 2. University of Utah, College of Social Work, Salt Lake City, UT, USA, 3. College of Social Work, School of Medicine, and Center for Alzheimer's Care Imaging and Research, University of Utah, Salt Lake City, UT, USA, 4. CNE, PhD Student, College of Nursing, University of Utah, Salt Lake City, UT, USA

Meaning-making - the capability of grievers to accept loss, realize growth, and reorganize personal identity contributes to healthy grief. We evaluated grief preparedness and grief experience in 50 family caregivers (FCGs) anticipating death of their care recipient (CR) within 6 months, and 50 FCGs who experienced the death of their CR in the prior 6 months. Using a phenomenological lens to discern meaning-making in narratives, we found that when preparation included positive memory construction, perception of death as ending CR suffering, relationship resolution, dementia understanding, and shared construction of meaning with family, it predicted positive grief. Inadequate preparation included trauma as CR declined, loss of caregiver role, perceived unavailability of support, loneliness and difficulties creating a life without CR. Inadequate preparation was associated with difficulty making meaning of the illness, death and poorer grief. These findings inform understanding of meaning-making as a mediating process in preparation and healthy grief.

\section{UTILISING THE ECOLOGICAL MODEL OF RESILIENCE TO UNDERSTAND VULNERABILITY IN OLDER WIDOWED}

K. Bennett, University of Liverpool, Liverpool, England, United Kingdom

Widowhood is a high-probability life event in later life. Whilst bereavement and subsequent widowhood are naturally highly stressful, the majority of widowed men and 